\title{
Stromal fibroblasts support dendritic cells to maintain IL-23/Th17 responses after
}

exposure to ionizing radiation

*Anna Malecka, ${ }^{*}$ Qunwei Wang, ${ }^{*}$ Sabaria Shah, ${ }^{\dagger}$ Ruhcha V Sutavani, ${ }^{\dagger}$ lan Spendlove,

†Judith M Ramage, " "Julie Greensmith, "Hester A Franks, §Michael J Gough, "Anja

Saalbach, "Poulam M Patel, ${ }^{* \#}$ Andrew M Jackson

*Host-Tumour Interactions Group \& ${ }^{\dagger}$ Cancer Immunotherapy Group, Division of Cancer \& Stem Cells, Nottingham, UK

${ }^{\ddagger}$ Cell Signalling and Immunology, University of Dundee

$\S$ Earle A Chiles Research Institute, Robert W Franz Cancer Center, Providence Portland Medical Center, Portland, OR, USA

K Klinik fur Dermatologie, University of Leipzig, Germany

"Intelligent Modelling and Analysis Research Group, University of Nottingham, UK

\section{Summary sentence}

Crosstalk between stromal fibroblasts and dendritic cells negates the immune suppressive effect of ionizing radiation.

Running Title: Dendritic cell-fibroblast crosstalk

\# Corresponding author

Host-Tumour Interactions Group, Division of Cancer \& Stem Cells, University of Nottingham, City Hospital, Hucknall Road, NG5 1PB, UK, +44 115 8231860, E:

Andrew.Jackson@nottingham.ac.uk 
Keywords: ionizing radiation, cytokine, immunity, cancer

Total character count: 25,042

Total number of figures: 5

Total number of color figures: 4

Total number of words in the Abstract: 233

Total number of words in the summary sentence: 14 


\section{Abbreviations:}

APC Antigen presenting cell, ATM Ataxia-telangiectasia mutated kinase, cAMP Cyclic adenosine 3',5'-monophosphate, CCL2 Chemokine (C-C Motif) Ligand 2, CCL20 Chemokine (C-C Motif) Ligand 20, COPD chronic obstructive pulmonary disease, COX2 cyclooxygenase 2, CXCL16 Chemokine (C-X-C Motif) Ligand 16, DC dendritic cell, DNAPK DNA-dependent protein kinase, FB fibroblast, iDC immature DC, IR ionizing radiation, LN lymph node, LPS lipopolysaccharide, mDC mature DC, MoDC monocyte-derived DC, PGE $_{2}$ prostaglandin E2, PKA Protein Kinase-A, PP2A Protein phosphatase 2A, rh recombinant human, TAF Tumor associated FB, TNFa tumor necrosis factor $\alpha$, TLR TollLike Receptor 


\section{Abstract}

Dendritic cell (DC) function is modulated by stromal cells including fibroblasts (FB). Whilst poorly understood, the signals delivered through this cross-talk substantially alter DC biology. This is well illustrated with release of TNF $\alpha /$ IL-1 $\beta$ from activated DC, promoting $\mathrm{PGE}_{2}$ secretion from stromal FB. This instructs DC to up-regulate IL-23, a key Th17polarising cytokine. We previously showed ionizing radiation (IR) inhibited IL-23 production by human DC in vitro. In the present study we investigated the hypothesis that DC-FB cross-talk overcomes the suppressive effect of IR to support appropriately polarized Th17 responses.

Radiation (1-6Gy) markedly suppressed IL-23 secretion by activated DC $(p<0.0001)$ without adversely impacting their viability, and consequently inhibited the generation of Th17 responses. Cytokine suppression by IR was selective as there was no effect on IL$1 \beta,-6,-10,-27$ or TNFa, and only a modest (11\%) decrease in IL-12p70 secretion. Coculture with FB augmented IL-23 secretion by irradiated DC and increased Th17 responses. Importantly, in contrast to DC, irradiated FB maintained their capacity to respond to $T N F \alpha / / L-1 \beta$ and produce $\mathrm{PGE}_{2}$, thus providing the key intermediary signals for successful DC-FB crosstalk.

In summary, stromal FB support Th17 polarizing cytokine production by DC that would otherwise be suppressed in an irradiated microenvironment. This has potential ramifications for understanding the immune response to local radiotherapy. These findings underscore the need to account for the impact of micro-environmental factors, including stromal cells, in understanding the control of immunity. 


\section{Introduction}

Cross-talk between DC and stromal FB has a marked impact on DC function with regards to their ability to mature, migrate, and trigger appropriate adaptive responses [1-3]. Whilst the importance of cross-talk between FB and the immune system for the onset and maintenance of cancer and autoimmunity was recently recognized, the exact mechanisms governing these interactions and the extent to which stromal cells affect the outcome of therapeutic interventions like radiotherapy remain poorly understood [4-7].

Fibroblasts play important roles in the pathology of a wide range of diseases. Tumor associated FB (TAF) promote tolerogenic DC in hepatic, breast and ovarian cancers while in pancreatic cancer they skew DC to promote Th2 responses [8-11]. Pulmonary FB are involved in the pathology of COPD (chronic obstructive pulmonary disease) and asthma by secreting CCL2 (Chemokine (C-C Motif) Ligand 2) and CCL20 (Chemokine (C-C Motif) Ligand 20) to attract DC to airways and thus maintain chronic inflammation [12]. Similarly, synovial FB are implicated in the perpetuation of rheumatoid arthritis through recruitment and activation of leucocytes including T cells, macrophages and DC $[7,13]$. Recently we established that FB modulate IL-23 secretion by DC to promote Th17 responses and this process was implicated in the maintenance and progression of psoriatic lesions [3]. This model proposes that dermal FB respond to TNF $\alpha$ (tumor necrosis factor $\alpha$ ) and IL-1 $\beta$, secreted from activated DC, by producing $\mathrm{PGE}_{2}$ (prostaglandin E2). Fibroblast-derived $\mathrm{PGE}_{2}$ acts in a juxtacrine manner to amplify IL-23 release from DC thus supporting the generation of Th17 responses. The IL-23/Th17 axis is important for the development and maintenance of autoimmune disorders including rheumatoid arthritis, psoriasis and colitis [14-17]. IL-23 promotes tumour growth directly and indirectly through Th17 responses that drive proliferation, invasion, metastasis and angiogenesis [18-23]. Furthermore, it is implicated in the development of idiopathic and radiation-induced fibrosis [24, 25]. 
Radiotherapy (RT) is a major tool for the treatment and palliation of tumours including squamous cell carcinoma of the skin, breast and primary brain tumours and brain metastases [26-28]. Current protocols employ fractionated RT typically comprising relatively low doses (1-6Gy) of ionising radiation (IR) administered over a period of weeks $[26,29]$ This allows tumour cells to be targeted with sufficient cumulative dose to deliver therapeutic benefit whilst restricting side effects to a tolerable minimum. However, during radiotherapy, immune and stromal cells residing in and adjacent to the tumour niche are also affected by IR through both direct and bystander effects. Irradiated tumour cells upregulate $\mathrm{MHC}$ I expression and secrete chemokines and cytokines such as CXCL16 (Chemokine (C-X-C Motif) Ligand 16), TNFa, IL-1 $\beta$ and IL-6 which in turn stimulate infiltration and activation of DC [30]. DC are relatively resistant to radiation-induced apoptosis maintaining viability at doses up to 30Gy [31]. Their radio-resistance is a result of constitutively expressed DNA repair systems including ATM (ataxia telangiectasia mutated) kinase and DNA-PK (DNA-dependent protein kinase) [32]. However, despite this, direct exposure of DC to IR suppresses their function. Irradiation of DC down-regulates the production of the $\mathrm{T}$ cell polarising cytokine IL-12 without affecting IL-10 thus changing the ratio between pro and anti-inflammatory stimuli and shifting the balance from $\mathrm{T}$ cell activation to tolerance [31]. Furthermore, we recently described that IR inhibits secretion of IL-23, another T-cell polarising cytokine, by DC [33]. Taken together this suggests that, in the setting of local radiotherapy, the cytokine "signal 3" from activated DC is substantially modified, thus altering the nature of subsequent T-cell responses.

We therefore investigated the hypothesis that local FB support IL-23 release by irradiated $\mathrm{DC}$ and thereby maintain their ability to generate Th17 responses. Doses of radiation consistent with those employed for fractionated RT, selectively inhibited IL-23 and to a lesser extent IL-12 by DC, without affecting IL-10, IL-6, IL-27, TNF $\alpha$ or IL-1 $\beta$ secretion. Interestingly, IR did not affect the capacity of FB to amplify IL-23 secretion by DC. The co- 
culture of irradiated FB with irradiated DC up-regulated IL-23 secretion and increased Th17 responses. We examined the factors by which FB support the function of DC despite the presence of ionizing radiation by employing a DC-FB co-culture system in the presence of IR. The enhancing effect of FB was mimicked by addition of $\mathrm{PGE}_{2}$ or forskolin to irradiated DC and was abrogated by treating FB with the COX2 (cyclooxygenase 2) inhibitor indomethacin. This effect occurred despite activation of the ATM pathway in irradiated DC which we previously established was involved in IL-23 down-regulation [33]. These findings indicate that even after exposure to ionizing radiation, activated FB act through secretion of $\mathrm{PGE}_{2}$ to activate the cAMP (Cyclic adenosine 3',5'-monophosphate) pathway in irradiated DC, leading to increased IL-23 secretion independent of ATM kinase. Our findings not only establish the significance of FB in regulating DC responses but also highlight the complex interplay between DC and their microenvironment, and illustrate the importance of developing more comprehensive cell biology models for understanding immunity. 


\section{Materials and methods}

\section{Reagents}

All reagents were endotoxin-free. Recombinant human GM-CSF and TNFa were from PeproTech, Rocky Hill, NJ; IL-4 and IFNy were from R\&D Systems Europe, Oxford, U.K.; Ultrapure TLR4-agonist (Salmonella Minnesota LPS) was from InvivoGen (San Diego, CA); Recombinant human IL-1 $\beta$ and IL-6 were from Immunotools, Friesoythe, Germany; IL-23 was from eBioscience (San Diego, CA); $\mathrm{PGE}_{2}$, Indomethacin and forskolin were from Sigma-Aldrich (Dorset, U.K.). Mouse anti-human CD4-PE was from BD Biosciences (Oxford, U.K.); mouse anti-human CD4-PECy7, mouse anti-human CD45RA-FITC, mouse anti-human CD14-Pe-Cy5.5 and matching isotype controls were from eBioscience (San Diego, CA). For CD4 activation, mouse anti-human CD28 was obtained from BD Biosciences, IL-2 (R\&D systems), and CD3 (OKT3) was produced in-house. AnnexinV/PI staining kit was obtained from BD Bioscience (Oxford, UK).

Generation of monocyte-derived DC

Monocyte-derived DC were generated as previously described [34]. Briefly, fresh blood samples were obtained from healthy volunteers, and buffy coats were obtained from the National Blood Transfusion Service in accordance with the approval of the relevant ethical review boards. PBMC were isolated using endotoxin-free Histopaque 1.077(Sigma Aldrich, Dorset, U.K.) gradient centrifugation. CD14 ${ }^{+}$monocytes were purified using anti-CD14 magnetic beads (Miltenyi Biotec, Bergisch Gladbach, Germany). DC were generated by culture in DC medium (RPMI 1640, 10\% fetal calf serum, $1 \%$ sodium pyruvate -all from Sigma Aldrich Dorset, U.K- containing recombinant human (rh) GM-CSF (1000 U/mL) and rhIL-4 (1000 U/mL) for 5 days. Additional complete medium was added on day 3 . The purity and quality of DC were determined by flow-cytometry and morphologic analysis. 
DC-FB co-culture

The human dermal FB cell line BJ6 was obtained from Dr. Lloyd Hamilton (University of Nottingham) whilst primary dermal FB were obtained from Dr. Anja Saalbach (University of

Leipzig). All cells were tested free of mycoplasma infection prior to use. For co-culture, FB were seeded in flat-bottomed 96 well plates and rested overnight. DC were added to FB at a 4:1 ratio. Co-cultures were incubated for $24 \mathrm{~h}$ in a humidified atmosphere of $5 \%$ $\mathrm{CO}_{2}$ in air at $37^{\circ} \mathrm{C}$. Supernatants were collected and stored at $-20^{\circ} \mathrm{C}$. All experimental conditions were performed in biological triplicates and on multiple donors. In some experiments the COX-2 inhibitor indomethacin $(2 \mu \mathrm{M})$ was added to FB prior to co-culture to determine the contribution of $\mathrm{PGE}_{2}$ synthesis in the induction of IL-23. For assessment of cell-cell interaction Costar Transwell permeable support system was used with FB in the lower and DC in the upper chamber separated by $3 \mu \mathrm{m}$ pores. Primary FB were used up to and including the $4^{\text {th }}$ passage after which they were discarded.

Irradiation of cells

Cells were irradiated in tissue culture plates immediately prior to DC activation with LPS (lipopolysaccharide) $(500 \mathrm{ng} / \mathrm{mL})$ and IFNy $(1000 \mathrm{U} / \mathrm{mL})$. For experiments with indomethacin, DC were activated $3 \mathrm{~h}$ before irradiation and adding to FB to minimize the effect of COX2 inhibitor on DC maturation. Cells were irradiated (0-6Gy of 195kVp X-rays, 0.87Gy/min, 0.5mm Cu filter, 48.4cm FSD) using a Gulmay Xstrahl cabinet irradiation system. Cell morphology was monitored by phase-contrast microscopy (x40) following IR and again after a further $24 \mathrm{~h}$ of culture. Cell viability, apoptosis and necrosis were determined $24 \mathrm{~h}$ after radiation by trypan blue exclusion and AnnexinV/PI FACS using 
DMSO as a positive control (not shown). Flow cytometry was performed using Beckmann Coulter FC500 flow cytometer and analyzed with FlowJo software.

\section{Measurement of secreted cytokine}

The secretion of IL-23p40/p19 or IL-12p70 was determined by commercial human IL-23 Ready-set-go ELISA (eBioscience, San Diego, CA) and human IL-12p70 ELISA kits (BD Biosciences, Oxford, U.K.). Assays did not significantly react with other proteins, and the sensitivities were 15 and $7.8 \mathrm{pg} / \mathrm{mL}$ respectively. IL-6 was measured by ELISA (ImmunoTools-Friesoythe, Germany) with a sensitivity of $9 \mathrm{pg} / \mathrm{mL}$. IL-1 $\beta$, TNFa, IL-17, IL27 and $\mathrm{PGE}_{2}$ were measured with DuoSet assays (R\&D Systems Europe, Oxford, U.K), and assay sensitivity was $3.9,15.6,7.8,156$ and $30.9 \mathrm{pg} / \mathrm{mL}$ respectively. Absorbance was measured at 450nM using a spectrophotometer.

\section{Generation of Th17 responses}

DC-FB co-cultures were treated as previously described for $12 \mathrm{~h}$ then washed with fresh medium to limit the impact of LPS/IFN $\gamma$ on T-cells. We previously established that IL-23 is not produced by DC until >12h after TLR (Toll-Like Receptor) stimulation [35]. Human naïve $C D 4^{+} T$ cells were obtained from fractionated fresh whole blood and naïve CD4 ${ }^{+}$ cells were obtained via a two-step isolation procedure using the Naïve CD4 ${ }^{+} \mathrm{T}$ Cell Isolation Kit (Miltenyi Biotec, Bergisch Gladbach, Germany). Isolated T cells $\left(C D 4^{+} \mathrm{CD}_{45 \mathrm{RA}^{+}}\right.$, purity> 95\%) were cultured in 48 well plates at $2 \times 10^{5}$ cells per well. Allogeneic T cells were cultured in a 1:1 ratio of fresh medium and DC: FB co-culture supernatant for 5 days in the presence of anti-CD3 (OKT3; $1 \mu \mathrm{g} / \mathrm{ml})$ and anti-CD28 $(5 \mu \mathrm{g} / \mathrm{ml})$ antibodies with IL-2 (50IU/ml). As controls for Th17 polarization and the 
importance of IL-23, T cells were treated with recombinant IL-1 $\beta$, IL-6 and IL-23. T cells were rested for 2 days and re-stimulated with anti-CD3/anti-CD28. For flow-cytometric assessment of Th17, cells were treated with Brefeldin A (BD Bioscience, Oxford, U.K.) for 20h, fixed with 0.5\% formaldehyde, permeabilized (Perm Buffer, Biolegend, San Diego, CA) and incubated with mouse anti-human IL-17 FITC (eBioscience San Diego, CA). Flow cytometry was performed using Beckman Coulter FC500 flow cytometer and analyzed with FlowJo software. Supernatants from parallel cultures without Brefeldin A were harvested after $48 \mathrm{~h}$ for ELISA.

Measurement of intracellular phospho-ATM

For measurement of intra-cellular phospho-ATM (pATM) co-cultures were separated using 0.3um membrane Transwell plates (Corning, NY, USA). FB were seeded in the lower chamber and allowed to rest overnight before DC were added to the upper chambers. Cultures were irradiated and immediately activated with LPS/IFNy. After 12h, DC were fixed in cold $2 \%$ formaldehyde/PBS and permeabilized with cold methanol. Binding of primary pATM (Ser1981) (eBioscience San Diego, CA) antibody was detected with a FITC-conjugated secondary Ab (Dako, Glostrup, Denmark). Cells were acquired using a MACSQuant cytometer and analyzed using FlowJo.

Quantitation of mRNA level for IL23A, IL12B with real-time RT-PCR

Real-time PCR was performed as previously described [33, 34]. In short cells were plated in DC medium, rested for $1 \mathrm{~h}$, irradiated and immediately stimulated with LPS/IFNy. RNA was isolated (Nucleospin RNAll kit, Macherey-Nagel Düren, Germany) and cDNA prepared using GoScript Reverse Transcription system, Promega Madison, WI. Taqman 
quantitative PCR was carried out for IL12B and IL23A with TOP1 as housekeeping gene (Applied Biosystems Paisley, UK, TOP1 Hs00243257_m1, Hs00168405_m1, IL-12B/IL12p40 Hs00233688_m1, IL-23A/IL-23p19 Hs00372324_m1), with Mastermix (Primer Design Southampton, UK) on a Stratagene MX3005P and analyzed with Stratagene software. Quantification was done by $\delta \delta \mathrm{CT}$ method where $\delta \mathrm{CT}=$ (gene of interest $\mathrm{CT})$ - (TOP1 CT), $\delta \delta C T$ calculated with $\mathrm{mDC}$ at 0Gy as reference condition.

\section{Statistical analysis}

Results were statistically analyzed using student's t-test or 2-way ANOVA in GraphPad ${ }^{\circledR}$ Prism software and figures annotated as follows: *:0.05>p $\geq 0.01,{ }^{* *}: 0.01 p \geq 0.001$, ${ }^{* * *}: 0.001>p \geq 0.0001,{ }^{* * *}: p<0.0001$. Results are presented as mean \pm standard deviation (SD). 


\section{Results}

Irradiated FB recover IL-23 secretion from irradiated DC

The impact of stromal cells on the response of DC to ionizing radiation has not previously been studied. Therefore, we tested the hypothesis that DC-FB cross-talk overcomes the immune-inhibitory effect of IR. We recently established that exposure of DC to IR inhibits TLR4-dependent IL-23 secretion through the activation of ATM kinase [33]. In agreement with this, irradiation of DC significantly suppressed TLR-dependent IL-23 secretion. Radiation induced suppression of IL-23 was dose-dependent and maximal at 6Gy $(p<0.001$ Figure 1A) and occurred in all donors tested $(p<0.05)$ (Figure 1B \& C, Table 1).

Co-culture of FB with TLR4-activated DC (mDC) markedly increased the secretion of IL-23 $(p<0.05$, Figure 1D). Furthermore, we determined the ability of irradiated FB to sustain IL23 responses from irradiated DC. Importantly, irradiated FB maintained their ability to support increased IL-23 secretion from irradiated DC $(p<0.01)$ in all donors (Figure 1D). The levels of IL-23 secreted by irradiated mDC co-cultured with irradiated FB were greater than or equal to those obtained from non-irradiated mDC controls. It should be noted that whilst IL-23 secretion was increased in the presence of FB, the levels were nevertheless lower than those obtained from non-irradiated co-cultures. However, the fold increase of IL-23 secretion by co-cultures was similar regardless of irradiation (Figure 1E). The ability of FB to increase IL-23 secretion by irradiated DC was maintained regardless of whether the BJ6 cell line or primary dermal FB were used (Figure 1F). As expected, neither immature DC (iDC) nor FB secreted IL-23 irrespective of irradiation status or addition of FB (data not shown).

In the presence of IL- 6 and IL-1 $\beta$, IL-23 serves a key role in the polarization of human naïve $\mathrm{CD}^{+} \mathrm{T}$ cells towards a Th17 phenotype $[33,36]$. Therefore, we determined whether the protective effect conferred by FB on IL-23 production resulted in the induction of Th17 
responses. Stimulation of naïve $\mathrm{CD} 4^{+} \mathrm{T}$ cells through $\mathrm{CD} 3 / \mathrm{CD} 28$ in the presence of conditioned supernatants from $\mathrm{mDC}$ cultures elicited Th17 responses as shown by secretion of IL-17A (Figure 1G). Furthermore, the addition of FB to DC enhanced IL-17 secretion by $T$ cells in all donors tested irrespective of exposure to IR.

Irradiated DC maintain their ability to stimulate fibroblasts

To determine the mechanism by which FB increase IL-23 secretion in irradiated DC we tested each step in the DC-FB co-culture. Initially we determined if the secretion of TNFa and IL-1 $\beta$ were reduced by IR [2]. In contrast to IL-23, DC maintained their secretion of TNF $\alpha$ and IL-1 $\beta$ following exposure to IR (Table 1).

In view of the differential effect of IR on cytokine expression we assessed the secretion of other cytokines including IL-12, IL-27, IL-6 and IL-10 (Table 1). The inhibitory effect of IR was restricted to IL-12 and IL-23 which share common p40 subunit. IR strongly downregulated IL-23 and only suppressed IL-12 to a modest extent. Interestingly another member of IL-12 family, IL-27, was unaffected. Production of the pro-inflammatory cytokine IL-6, as well as anti-inflammatory IL-10 were unaffected by IR. Next we determined whether IR affected transcription of the IL-23-specific IL-23A gene or the common $I L-12 B$ gene that encodes the p40 chain. Irradiation of DC (6Gy) significantly suppressed transcription of $I L-23 A$ when compared with un-irradiated $D C(p<0.0001)$. Although the effect of IR on IL-12B was also inhibitory it was nevertheless less marked than on IL-23A (Figure 2A, B).

In contrast to many other cell types (e.g. monocytes), DC remain viable when irradiated [37]. This is because DC (but not classical monocytes) have constitutively active DNArepair systems that repair double-strand DNA-breaks [38]. We therefore confirmed that 
the suppression of IL-23 we observed in irradiated DC was not due to apoptosis or necrosis. lonizing radiation did not impact on the viability of DC. Staining with AnnexinV/PI showed the viability of DC to be to be unaffected by IR (Figure $2 \mathrm{C}$ ).

FB are functionally unimpaired by IR

Since irradiated DC retained their ability to secrete TNFa/IL1 $\beta$ we next investigated the effect of IR on the response of FB to these cytokines. Co-culture of irradiated FB with nonirradiated DC did not impair their capacity to promote IL-23 release from mDC (Figure 3A, B). Furthermore, irradiated FB retained their ability to respond to signals received from DC and secrete $\mathrm{PGE}_{2}$ since stimulation of irradiated BJ6 and primary FB with exogenous TNF $\alpha /$ IL-1 $1 \beta$ elicited $\mathrm{PGE}_{2}$ secretion which was unaffected by radiation (Figure 3C, D). As expected, exposure of resting FB to radiation did not elicit cytokine release. In the absence of DC, FB did not secrete IL-6, IL-12, IL-10, IL-17, IL-23 or IL-27 in response to activation with TNFa, IL-1 $1 \beta$ or LPS/IFNy irrespective of exposure to IR (data not shown). Irradiation had no impact on FB viability as assessed by dye-exclusion (data not shown) and AnnexinV/PI staining (Figure 3E). It is important to stress that we examined the effects of IR on FB within the first $24 \mathrm{~h}$ of exposure after which time activated DC would be expected to migrate to regional LN [39].

FB support function of irradiated DC through COX2-dependent $P G E_{2}$ release

Previously we established the importance of FB for IL-23 secretion by DC and demonstrated that this was mediated through COX2-dependent PGE 2 [3]. To assess whether this mechanism was involved in irradiated cells we first demonstrated the involvement of FB-derived soluble factors. FB were separated from DC by a $0.3 \mu \mathrm{m}$ 
porous membranes in Transwell plates. Despite the lack of cell-cell contact, FB retained their capacity to support IL-23 secretion from irradiated DC (Figure 4A). Therefore, we evaluated the involvement of $\mathrm{PGE}_{2}$ in up-regulation of IL-23 secretion by irradiated DC. The addition of $\mathrm{PGE}_{2}$ to $\mathrm{DC}$ immediately after irradiation and activation resulted in a significant increase of IL-23 secretion in all donors tested (Figure 4B). Because PGE 2 secretion by FB is usually COX2 dependent $[2,37]$ we determined the importance of COX2 activation for FB-dependent recovery of IL-23 secretion using the COX2 inhibitor, indomethacin. IL-23 production from irradiated co-cultures was significantly reduced by indomethacin (Figure 4C). In the presence of indomethacin, IL-23 secretion from DC-FB co-cultures was reduced to levels similar to those achieved in the absence of FB.

FB promote irradiated DC IL-23 responses through the CAMP pathway Irradiation of human DC mono-cultures selectively inhibits IL-23 by phosphorylation of the ATM kinase [33]. However $\mathrm{PGE}_{2}$ also up-regulates IL-23 through activation of the cAMPPKA (Protein Kinase-A) signaling pathway [40, 41]. Interestingly, in lung cancer cAMP signaling inhibits IR-induced phosphorylation of ATM [42]. Therefore, we sought to dissect the molecular mechanism responsible for IL-23 secretion in irradiated DC-FB co-cultures.

We initially assessed ATM activation [3] in irradiated DC mono-cultures or DC-FB cocultures. DC-FB co-cultures were separated by $0.3 \mu \mathrm{m}$ porous membrane to ensure that phospho-ATM (pATM) levels were measured only in DC. As expected, irradiation of mDC resulted in ATM phosphorylation after $2 \mathrm{~h}$ and this persisted for at least $12 \mathrm{~h}$ as demonstrated by intracellular staining with flow cytometry (Figure 4D). However co-culture with FB did not suppress the levels of ATM expressed by irradiated DC suggesting that the support of DC function by FB occurred independently of ATM kinase. Lastly, we examined the involvement of the cAMP pathway in regulation of IL-23 from irradiated DC. 
The addition of the cAMP active analogue Forskolin to irradiated mono-cultures of $\mathrm{mDC}$ resulted in a dose-dependent increase in IL-23 $(p<0.01)$ (Figure 4E), implicating a role for the cAMP signal transduction pathway. 


\section{Discussion}

Studies on the impact of the stromal microenvironment on immunity are important as immune cells are in constant cross-talk with their stroma during each and every stage of the immune response. Stromal cells have the ability to affect a wide range of immune functions including DC maturation, their migration to lymph nodes, and subsequent polarization of $\mathrm{T}$ cell responses $[1,2,12,43]$. The present study investigated the ability of stroma to modulate the outcome of therapeutic interventions directed towards immune system. Our previous work established that IR inhibits the cytokine response of DC and in particular IL-23 [33]. On the other hand, stromal FB support IL-23 production by activated DC [2]. Therefore, we addressed the hypothesis that FB, cells which are known to be relatively radio-resistant [44], continue to support the function of DC in the presence of IR.

The doses of IR used in the current study are similar to those employed during routine radiotherapy of common malignancies $[26,29]$. At these doses IR damages transformed cells and initiates their demise by generating free radicals which induce stress responses and consequently cell death if the damage is not repaired [29, 38, 45, 46]. Due to their high proliferative rates and impaired DNA repair mechanisms many tumor types are selectively sensitive to radiation-induced DNA damage [30, 47]. However, whilst tumor cells are the main target for radiotherapy, immune and stromal cells residing in the tumor microenvironment are also exposed to and affected by IR [48]. In this regard, previous work using mono-cultures has shown that IR suppresses IL-12 secretion by moDC between 2 and 20Gy [31] while another report showed increased IL-12 secretion by murine DC at $0.05 \mathrm{~Gy}$ and this effect was reversed to the level of non-irradiated DC at $1 \mathrm{~Gy}$ [49]. The disparity between these findings may be caused by differences in the behavior of human and murine DC however they may also reflect different mechanisms of action for 
IR at extremely low doses (0.05-1Gy) comparing to higher doses used for radiotherapy (2$6 \mathrm{~Gy})[26,50]$.

We found that the inhibitory effect of IR was restricted to IL-12 family members sharing the common p40 subunit. However, the effect was considerably more pronounced for IL-23 than IL-12p70. Interestingly the maximal effect of IR was exerted at $6 \mathrm{~Gy}$ with a plateau of effect at higher doses. This may be due to ATM reaching its maximum activation state at this dose. On the other hand, the effect of IR did not extend to the other IL-12 family member, IL-27. IL-27 comprises the IL-27p28 subunit and Epstein-Barr virus-induced gene 3 (EBI3) which are related to p35 and p40 respectively [51]. Those findings demonstrate the highly selective effect of IR on DC functions with prevalence to inhibit Th17 responses. The lack of effect of IR on TNF $\alpha$ and IL-1 $\beta$ had important consequences for our multicellular model as it allowed activated DC to sustain their interactions with FB and generate the $\mathrm{PGE}_{2}$ feedback loop [3]. Irradiated FB also continued to support the production of IL-23 by DC irrespective of the dose of IR to which FB were exposed. This was observed not only with a FB cell line, but also with primary dermal FB thus supporting the potential physiological importance of this observation.

Fibroblasts (resting, TLR-activated, or irradiated) were not responsible for the secretion of any of the cytokines affected by IR. Previous reports describe FB expression of the IL23p19 subunit upon stimulation with IL-1 [52] but to the best of our knowledge there are no reports of IL-23 heterodimer secretion [53]. It is important to recognize the difference between biologically active IL-23 heterodimer secreted by APC, and IL-23p19 monomer and in the current study we measured heterodimeric IL-23 secretion [53].

It is known that FB can enter a senescent phase when exposed to IR yet remain viable $[18,44,54]$. This represents one of the important mechanisms of tumor development as in this state senescent FB alter their phenotype and promote tumor growth, invasion and 
render adjacent tumor cells increasingly radio-resistant [44]. Therefore, we assessed the effect of IR on FB in functional assays. $\mathrm{PGE}_{2}$ stimulates IL-23 secretion in human and murine DC $[2,41,55,56]$. Previous reports have published conflicting data in regard to regulation of $P G E_{2}$ secretion by gingival $F B$ irradiated with low-level diode laser $[57,58]$. In our model, irradiation of dermal FB did not alter $\mathrm{PGE}_{2}$ secretion in response to exogenous TNF $\alpha$ and IL-1 $\beta$, and this likely accounts for their continued capacity to augment IL-23 release by non-irradiated DC. Therefore, irradiated FB were unimpaired in their ability to secrete soluble immune modulators and maintained their capacity to respond to environmental stimuli.

According to recent reports $\mathrm{PGE}_{2}$ stimulates IL-23 secretion from DC through activation of the cAMP/PKA pathway $[40,41]$ and we previously described that IR down-regulates IL23 through phosphorylation of ATM kinase [33]. Interestingly, cAMP signaling inhibits radiation induced phosphorylation of ATM in lung cancer [42]. Addition of forskolin increased IL-23 secretion by irradiated DC to levels commensurate with those from irradiated DC-FB co-cultures, suggesting a role for CAMP. However, in contrast to Cho and colleagues, co-culture of irradiated DC with FB did not affect pATM levels despite upregulated IL-23. This disparity may be due to differences in timing of cAMP and ATM activation. Cho and co-workers activated cAMP before irradiation of cells thus preventing phosphorylation of ATM by activated PP2A (Protein phosphatase 2A). In our study DC were irradiated before activation and subsequent stimulation from FB. Since ATM is phosphorylated in DC within 15min of IR [33], signals from FB are received by DC too late to inhibit ATM activation. It is therefore even more notable that up-regulation of IL-23 secretion by FB occurred despite ATM activation.

The impact of FB on the ability of irradiated DC to promote Th17 responses was examined. Previously we and others established the role of IL-23 in the generation of 
Th17 [2, 33, 56]. In agreement with this, IL-17 secretion from T-cells conditioned with supernatants from DC-FB co-cultures was enhanced as compared to supernatants from $\mathrm{DC}$ mono-culture. $\mathrm{PGE}_{2}$ was shown to promote Th17 directly and in conjunction with IL-23 $[59,60]$. In our model the concentration of $\mathrm{PGE}_{2}$ secreted by FB was unaffected by IR. On the other hand, changes in IL-17 reflected differences in IL-23 levels, demonstrating the biological importance of FB-dependent IL-23 secretion in regulation of adaptive immunity. In summary we show that although IR inhibits IL-23 secretion from DC mono-cultures, the inclusion of FB provides a positive feedback loop that serves to maintain IL-23 secretion by DC. We propose that this translates to enhanced Th17 responses in a post-RT environment (Fig 5) and studies to investigate this are underway. Since IL-17 is a critical factor driving post-radiation fibrosis [24] this work identifies a potential mechanism for the pathological consequences of radiation and chemotherapy [25,61]. Importantly it highlights the need for multicellular models of the immune microenvironment. Insight into the complex interactions between the immune system and stroma is necessary to our understanding of pathology and for development of novel therapeutic interventions. 


\section{References}

1. Saalbach, A., Klein, C., Sleeman, J., Sack, U., Kauer, F., Gebhardt, C., Averbeck, M., Anderegg, U., Simon, J. C. (2007) Dermal fibroblasts induce maturation of dendritic cells. J Immunol 178, 4966-74.

2. Saalbach, A., Klein, C., Schirmer, C., Briest, W., Anderegg, U., Simon, J. C. (2010) Dermal fibroblasts promote the migration of dendritic cells. J Invest Dermatol 130, 444-54.

3. Schirmer, C., Klein, C., von Bergen, M., Simon, J. C., Saalbach, A. (2010) Human fibroblasts support the expansion of IL-17-producing T cells via up-regulation of IL23 production by dendritic cells. Blood 116, 1715-25.

4. Comito, G., Giannoni, E., Segura, C. P., Barcellos-de-Souza, P., Raspollini, M. R., Baroni, G., Lanciotti, M., Serni, S., Chiarugi, P. (2014) Cancer-associated fibroblasts and M2-polarized macrophages synergize during prostate carcinoma progression. Oncogene 33, 2423-31.

5. Chiarugi, P. (2013) Cancer-associated fibroblasts and macrophages: Friendly conspirators for malignancy. Oncoimmunology 2, e25563.

6. Yarnold, J. and Brotons, M. C. (2010) Pathogenetic mechanisms in radiation fibrosis. Radiother Oncol 97, 149-61.

7. Komatsu, N., Okamoto, K., Sawa, S., Nakashima, T., Oh-hora, M., Kodama, T., Tanaka, S., Bluestone, J. A., Takayanagi, H. (2014) Pathogenic conversion of Foxp3+ T cells into TH17 cells in autoimmune arthritis. Nat Med 20, 62-8.

8. De Monte, L., Reni, M., Tassi, E., Clavenna, D., Papa, I., Recalde, H., Braga, M., Di Carlo, V., Doglioni, C., Protti, M. P. (2011) Intratumor T helper type 2 cell infiltrate correlates with cancer-associated fibroblast thymic stromal lymphopoietin production and reduced survival in pancreatic cancer. J Exp Med 208, 469-78. 
9. Gobert, M., Treilleux, I., Bendriss-Vermare, N., Bachelot, T., Goddard-Leon, S., Arfi, V., Biota, C., Doffin, A. C., Durand, I., Olive, D., Perez, S., Pasqual, N., Faure, C., Ray-Coquard, I., Puisieux, A., Caux, C., Blay, J. Y., Menetrier-Caux, C. (2009) Regulatory T cells recruited through CCL22/CCR4 are selectively activated in lymphoid infiltrates surrounding primary breast tumors and lead to an adverse clinical outcome. Cancer Res 69, 2000-9.

10. Fialova, A., Partlova, S., Sojka, L., Hromadkova, H., Brtnicky, T., Fucikova, J., Kocian, P., Rob, L., Bartunkova, J., Spisek, R. (2013) Dynamics of T-cell infiltration during the course of ovarian cancer: the gradual shift from a Th17 effector cell response to a predominant infiltration by regulatory T-cells. Int J Cancer 132, 1070 9.

11. Bhatt, A., Curbishley, S. M., Houghton, E. L., Weston, C. J., Blahova, M., Adams, D. H. (2010) The Stromal Compartment of Hepatocellular Carcinoma Promotes the Local Differentiation of Tolerogenic Dendritic Cells. Hepatology 52, 344a-344a.

12. Kitamura, H., Cambier, S., Somanath, S., Barker, T., Minagawa, S., Markovics, J., Goodsell, A., Publicover, J., Reichardt, L., Jablons, D., Wolters, P., Hill, A., Marks, J. D., Lou, J., Pittet, J. F., Gauldie, J., Baron, J. L., Nishimura, S. L. (2011) Mouse and human lung fibroblasts regulate dendritic cell trafficking, airway inflammation, and fibrosis through integrin alphavbeta8-mediated activation of TGF-beta. J Clin Invest 121, 2863-75.

13. Huber, L. C., Distler, O., Tarner, I., Gay, R. E., Gay, S., Pap, T. (2006) Synovial fibroblasts: key players in rheumatoid arthritis. Rheumatology (Oxford) 45, 669-75.

14. Langrish, C. L., Chen, Y., Blumenschein, W. M., Mattson, J., Basham, B., Sedgwick, J. D., McClanahan, T., Kastelein, R. A., Cua, D. J. (2005) IL-23 drives a pathogenic $\mathrm{T}$ cell population that induces autoimmune inflammation. J Exp Med 201, 233-40. 
15. Tang, C., Chen, S., Qian, H., Huang, W. (2012) Interleukin-23: as a drug target for autoimmune inflammatory diseases. Immunology 135, 112-24.

16. Yannam, G. R., Gutti, T., Poluektova, L. Y. (2012) IL-23 in infections, inflammation, autoimmunity and cancer: possible role in HIV-1 and AIDS. J Neuroimmune Pharmacol 7, 95-112.

17. Liu, F. L., Chen, C. H., Chu, S. J., Chen, J. H., Lai, J. H., Sytwu, H. K., Chang, D. M. (2007) Interleukin (IL)-23 p19 expression induced by IL-1beta in human fibroblast-like synoviocytes with rheumatoid arthritis via active nuclear factorkappaB and AP-1 dependent pathway. Rheumatology (Oxford) 46, 1266-73.

18. Suzuki, H., Ogawa, H., Miura, K., Haneda, S., Watanabe, K., Ohnuma, S., Sasaki, H., Sase, T., Kimura, S., Kajiwara, T., Komura, T., Toshima, M., Matsuda, Y., Shibata, C., Sasaki, I. (2012) IL-23 directly enhances the proliferative and invasive activities of colorectal carcinoma. Oncol Lett 4, 199-204.

19. Langowski, J. L., Zhang, X., Wu, L., Mattson, J. D., Chen, T., Smith, K., Basham, B., McClanahan, T., Kastelein, R. A., Oft, M. (2006) IL-23 promotes tumour incidence and growth. Nature 442, 461-5.

20. Wang, L., Yi, T., Kortylewski, M., Pardoll, D. M., Zeng, D., Yu, H. (2009) IL-17 can promote tumor growth through an IL-6-Stat3 signaling pathway. J Exp Med 206, 1457-64.

21. He, D., Li, H., Yusuf, N., Elmets, C. A., Li, J., Mountz, J. D., Xu, H. (2010) IL-17 promotes tumor development through the induction of tumor promoting microenvironments at tumor sites and myeloid-derived suppressor cells. J Immunol $184,2281-8$

22. Alexandrakis, M. G., Pappa, C. A., Miyakis, S., Sfiridaki, A., Kafousi, M., Alegakis, A., Stathopoulos, E. N. (2006) Serum interleukin-17 and its relationship to angiogenic factors in multiple myeloma. Eur J Intern Med 17, 412-6. 
23. Zhu, X., Mulcahy, L. A., Mohammed, R. A., Lee, A. H., Franks, H. A., Kilpatrick, L., Yilmazer, A., Paish, E. C., Ellis, I. O., Patel, P. M., Jackson, A. M. (2008) IL-17 expression by breast-cancer-associated macrophages: IL-17 promotes invasiveness of breast cancer cell lines. Breast Cancer Res 10, R95.

24. Wang, B. Z., Wang, L. P., Han, H., Cao, F. L., Li, G. Y., Xu, J. L., Wang, X. W., Wang, L. X. (2014) Interleukin-17A antagonist attenuates radiation-induced lung injuries in mice. Exp Lung Res 40, 77-85.

25. Gasse, P., Riteau, N., Vacher, R., Michel, M. L., Fautrel, A., di Padova, F., Fick, L., Charron, S., Lagente, V., Eberl, G., Le Bert, M., Quesniaux, V. F., Huaux, F., Leitede-Moraes, M., Ryffel, B., Couillin, I. (2011) IL-1 and IL-23 mediate early IL-17A production in pulmonary inflammation leading to late fibrosis. PLoS One 6, e23185.

26. Overgaard, J., Hansen, H. S., Specht, L., Overgaard, M., Grau, C., Andersen, E., Bentzen, J., Bastholt, L., Hansen, O., Johansen, J., Andersen, L., Evensen, J. F. (2003) Five compared with six fractions per week of conventional radiotherapy of squamous-cell carcinoma of head and neck: DAHANCA 6 and 7 randomised controlled trial. Lancet 362, 933-40.

27. Pierce, S. M., Recht, A., Lingos, T. I., Abner, A., Vicini, F., Silver, B., Herzog, A., Harris, J. R. (1992) Long-term radiation complications following conservative surgery (CS) and radiation therapy (RT) in patients with early stage breast cancer. Int J Radiat Oncol Biol Phys 23, 915-23.

28. Gaspar, L. E., Mehta, M. P., Patchell, R. A., Burri, S. H., Robinson, P. D., Morris, R. E., Ammirati, M., Andrews, D. W., Asher, A. L., Cobbs, C. S., Kondziolka, D., Linskey, M. E., Loeffler, J. S., McDermott, M., Mikkelsen, T., Olson, J. J., Paleologos, N. A., Ryken, T. C., Kalkanis, S. N. (2010) The role of whole brain radiation therapy in the management of newly diagnosed brain metastases: a 
systematic review and evidence-based clinical practice guideline. J Neurooncol 96, 17-32.

29. Lee, Y., Auh, S. L., Wang, Y., Burnette, B., Wang, Y., Meng, Y., Beckett, M., Sharma, R., Chin, R., Tu, T., Weichselbaum, R. R., Fu, Y. X. (2009) Therapeutic effects of ablative radiation on local tumor require CD8+ T cells: changing strategies for cancer treatment. Blood 114, 589-95.

30. Teitz-Tennenbaum, S., Li, Q., Davis, M., Chang, A. E. (2004) Mechanisms involved in radiation enhancement of intratumoral dendritic cell therapy. Ann Surg Oncol 11, S69-S69.

31. Merrick, A., Errington, F., Milward, K., O'Donnell, D., Harrington, K., Bateman, A., Pandha, H., Vile, R., Morrison, E., Selby, P., Melcher, A. (2005) Immunosuppressive effects of radiation on human dendritic cells: reduced IL-12 production on activation and impairment of naive T-cell priming. Br J Cancer 92, 1450-8.

32. Bauer, M., Goldstein, M., Christmann, M., Becker, H., Heylmann, D., Kaina, B. (2011) Human monocytes are severely impaired in base and DNA double-strand break repair that renders them vulnerable to oxidative stress. Proc Natl Acad Sci U S A 108, 21105-10.

33. Wang, Q., Franks, H. A., Lax, S. J., El Refaee, M., Malecka, A., Shah, S., Spendlove, I., Gough, M. J., Seedhouse, C., Madhusudan, S., Patel, P. M., Jackson, A. M. (2013) The ataxia telangiectasia mutated kinase pathway regulates IL-23 expression by human dendritic cells. J Immunol 190, 3246-55.

34. Franks, H. A., Wang, Q., Lax, S. J., Collins, M. K., Escors, D., Patel, P. M., Jackson, A. M. (2014) Novel function for the p38-MK2 signaling pathway in circulating $\mathrm{CD} 1 \mathrm{C}+(\mathrm{BDCA}-1+)$ myeloid dendritic cells from healthy donors and 
advanced cancer patients; inhibition of p38 enhances IL-12 whilst suppressing IL10. Int J Cancer $134,575-86$.

35. Jackson, A. M., Mulcahy, L. A., Porte, J., Franks, H. A., El Refaee, M., Wang, Q., Shah, S., Zhu, X., Patel, P. M. (2010) Role of mitogen-activated protein kinase and PI3K pathways in the regulation of IL-12-family cytokines in dendritic cells and the generation of T H-responses. Eur Cytokine Netw 21, 319-28.

36. Wang, Q., Franks, H. A., Porte, J., El Refaee, M., Shah, S., Crooks, J., Patel, P. M., Jackson, A. M. (2011) Novel approach for interleukin-23 up-regulation in human dendritic cells and the impact on T helper type 17 generation. Immunology 134, 6072.

37. Bukata, S. V., Gelinas, J., Wei, X., Rosier, R. N., Puzas, J. E., Zhang, X., Schwarz, E. M., Song, X. Y., Griswold, D. E., O'Keefe, R. J. (2004) PGE2 and IL-6 production by fibroblasts in response to titanium wear debris particles is mediated through a Cox-2 dependent pathway. J Orthop Res 22, 6-12.

38. Bauer, M., Goldstein, M., Heylmann, D., Kaina, B. (2012) Human monocytes undergo excessive apoptosis following temozolomide activating the ATM/ATR pathway while dendritic cells and macrophages are resistant. PLoS One 7, e39956.

39. Itano, A. A. and Jenkins, M. K. (2003) Antigen presentation to naive CD4 T cells in the lymph node. Nat Immunol 4, 733-9.

40. Kocieda, V. P., Adhikary, S., Emig, F., Yen, J. H., Toscano, M. G., Ganea, D. (2012) Prostaglandin E2-induced IL-23p19 subunit is regulated by cAMPresponsive element-binding protein and C/AATT enhancer-binding protein beta in bone marrow-derived dendritic cells. J Biol Chem 287, 36922-35.

41. Shi, Q., Yin, Z., Zhao, B., Sun, F., Yu, H., Yin, X., Zhang, L., Wang, S. (2015) PGE2 Elevates IL-23 Production in Human Dendritic Cells via a cAMP Dependent Pathway. Mediators Inflamm 2015, 984690. 
42. Cho, E. A., Kim, E. J., Kwak, S. J., Juhnn, Y. S. (2014) cAMP signaling inhibits radiation-induced ATM phosphorylation leading to the augmentation of apoptosis in human lung cancer cells. Mol Cancer 13, 36.

43. Sugita, K., Kabashima, K., Atarashi, K., Shimauchi, T., Kobayashi, M., Tokura, Y. (2007) Innate immunity mediated by epidermal keratinocytes promotes acquired immunity involving Langerhans cells and T cells in the skin. Clin Exp Immunol 147, 176-83.

44. Papadopoulou, A. and Kletsas, D. (2011) Human lung fibroblasts prematurely senescent after exposure to ionizing radiation enhance the growth of malignant lung epithelial cells in vitro and in vivo. Int J Oncol 39, 989-99.

45. Shiao, S. L. and Coussens, L. M. (2010) The tumor-immune microenvironment and response to radiation therapy. J Mammary Gland Biol Neoplasia 15, 411-21.

46. Barcellos-Hoff, M. H., Park, C., Wright, E. G. (2005) Radiation and the microenvironment - tumorigenesis and therapy. Nat Rev Cancer 5, 867-75.

47. Teitz-Tennenbaum, S., Li, Q., Okuyama, R., Davis, M. A., Sun, R., Whitfield, J., Knibbs, R. N., Stoolman, L. M., Chang, A. E. (2008) Mechanisms involved in radiation enhancement of intratumoral dendritic cell therapy. J Immunother 31, 34558.

48. Crittenden, M. R., Savage, T., Cottam, B., Baird, J., Rodriguez, P. C., Newell, P., Young, K., Jackson, A. M., Gough, M. J. (2014) Expression of arginase I in myeloid cells limits control of residual disease after radiation therapy of tumors in mice. Radiat Res 182, 182-90.

49. Shigematsu, A., Adachi, Y., Koike-Kiriyama, N., Suzuki, Y., Iwasaki, M., Koike, Y., Nakano, K., Mukaide, H., Imamura, M., Ikehara, S. (2007) Effects of low-dose irradiation on enhancement of immunity by dendritic cells. J Radiat Res 48, 51-5. 
50. Mestas, J. and Hughes, C. C. (2004) Of mice and not men: differences between mouse and human immunology. J Immunol 172, 2731-8.

51. Rousseau, F., Basset, L., Froger, J., Dinguirard, N., Chevalier, S., Gascan, H. (2010) IL-27 structural analysis demonstrates similarities with ciliary neurotrophic factor (CNTF) and leads to the identification of antagonistic variants. Proc Natl Acad Sci U S A 107, 19420-5.

52. Zhu, L., Wu, Y., Wei, H., Yang, S., Zhan, N., Xing, X., Peng, B. (2012) Upregulation of IL-23 p19 expression in human periodontal ligament fibroblasts by IL1beta via concurrent activation of the NF-kappaB and MAPKs/AP-1 pathways. Cytokine 60, 171-8.

53. Brentano, F., Ospelt, C., Stanczyk, J., Gay, R. E., Gay, S., Kyburz, D. (2009) Abundant expression of the interleukin (IL)23 subunit p19, but low levels of bioactive IL23 in the rheumatoid synovium: differential expression and Toll-like receptor-(TLR) dependent regulation of the IL23 subunits, $\mathrm{p} 19$ and $\mathrm{p} 40$, in rheumatoid arthritis. Ann Rheum Dis 68, 143-50.

54. Di Leonardo, A., Linke, S. P., Clarkin, K., Wahl, G. M. (1994) DNA damage triggers a prolonged p53-dependent G1 arrest and long-term induction of Cip1 in normal human fibroblasts. Genes Dev 8, 2540-51.

55. Sheibanie, A. F., Tadmori, I., Jing, H., Vassiliou, E., Ganea, D. (2004) Prostaglandin E2 induces IL-23 production in bone marrow-derived dendritic cells. FASEB J 18, 1318-20.

56. Qian, X., Gu, L., Ning, H., Zhang, Y., Hsueh, E. C., Fu, M., Hu, X., Wei, L., Hoft, D. F., Liu, J. (2013) Increased Th17 cells in the tumor microenvironment is mediated by IL-23 via tumor-secreted prostaglandin E2. J Immunol 190, 5894-902.

57. Pourzarandian, A., Watanabe, H., Ruwanpura, S. M., Aoki, A., Noguchi, K., Ishikawa, I. (2005) Er:YAG laser irradiation increases prostaglandin E production 
via the induction of cyclooxygenase-2 mRNA in human gingival fibroblasts. $\mathrm{J}$ Periodontal Res 40, 182-6.

58. Sakurai, Y., Yamaguchi, M., Abiko, Y. (2000) Inhibitory effect of low-level laser irradiation on LPS-stimulated prostaglandin E2 production and cyclooxygenase-2 in human gingival fibroblasts. Eur J Oral Sci 108, 29-34.

59. Chizzolini, C., Chicheportiche, R., Alvarez, M., de Rham, C., Roux-Lombard, P., Ferrari-Lacraz, S., Dayer, J. M. (2008) Prostaglandin E2 synergistically with interleukin-23 favors human Th17 expansion. Blood 112, 3696-703.

60. Yao, C., Sakata, D., Esaki, Y., Li, Y., Matsuoka, T., Kuroiwa, K., Sugimoto, Y., Narumiya, S. (2009) Prostaglandin E2-EP4 signaling promotes immune inflammation through Th1 cell differentiation and Th17 cell expansion. Nat Med 15, $633-40$.

61. Wilson, M. S., Madala, S. K., Ramalingam, T. R., Gochuico, B. R., Rosas, I. O., Cheever, A. W., Wynn, T. A. (2010) Bleomycin and IL-1beta-mediated pulmonary fibrosis is IL-17A dependent. J Exp Med 207, 535-52. 


\section{Authorship}

AM undertook or supervised all experimental work, interpreted the data and wrote the manuscript.

QW, SS, RVS and HAF undertook specific components of experimental work.

AS generated and characterized primary dermal fibroblasts and wrote the manuscript.

IS, JMR, JG and MJG undertook statistical analysis and wrote the manuscript.

MJG provided advice on radiation responses.

PMP conceived the original project idea and supervised the research.

AMJ conceived the original project idea, supervised the research and wrote the manuscript. 


\section{Acknowledgements}

This work was generously funded by grants from the MRC (HAF was funded by an RCUK MRC Clinical Research Training Fellowship, G0900336), The EPSRC (JG is in receipt of an EPSRC Discipline Hopping Award, EP/N007115/1), Matt's Trust for Cancer Research and the University of Nottingham (AM is in receipt of a Vice Chancellor's Research Excellence Scholarship). The authors are grateful to Dr. Bruno Gran (University of Nottingham) for his critical evaluation of the research leading to this manuscript and Dr. Lloyd Hamilton for provision of BJ6 fibroblasts. 


\section{Conflict of interests disclosure}

The authors declare no conflict of interest. 


\section{Figure legends}

Figure 1. FB promote IL23-Th17 axis during irradiation. IR suppresses IL-23. DC were irradiated prior to addition of LPS/IFNy and IL-23 release measured by ELISA; $\mathbf{A}-$ IL-23 suppression was dependent on the dose of IR (a representative donor from 3). B- shows a representative experiment at $6 \mathrm{~Gy}$ and $\mathbf{C}$ - shows a summary of 18 donors. D - FB rescue IL-23 secretion by irradiated DC. Co-culture of irradiated FB with irradiated DC upregulated IL-23 secretion (summary of 8 donors). E - shows fold change of IL-23 secretion by irradiated DC/FB co-cultures at 0 and 6Gy. F - Primary dermal FB demonstrate similar IL-23-enhancing activity to the BJ6 cell line (data from a representative donor or 5). G - FB permit irradiated DC to promote Th17 responses. IL17A secretion by naïve $C D 4^{+} \mathrm{T}$ cells activated with anti-CD3/anti-CD28 in the presence of supernatants of the indicated DC/FB cultures. T-cells were stimulated for 5 days and IL17A secretion determined after re-stimulation (collective data from 3 donors). Error bars indicate standard deviation (SD) of triplicate experiments.

Table 1. Effect of IR on cytokine secretion by mDC. DC were irradiated (6Gy) or nonirradiated before activation with LPS/IFNY. Supernatants were collected following $24 \mathrm{~h}$ incubation and secreted IL-23, IL-12, IL-27, IL-1ß, TNF $\alpha$, IL-6 and IL-10 were measured by ELISA. All experiments were repeated at least 4 times in triplicate and significance was assessed with 2-way ANOVA.

Figure 2. Effect of IR on DC. Changes in IL-23 secretion were associated with decreased transcription of the; $\mathbf{A}-I L-23 A$ gene and $\mathbf{B}-I L-12 B$ gene as shown using Q-RT-PCR in 3 donors. Statistical significance was measured by the student $t$-test. C - Despite exposure 
to up to 6Gy IR, there was no decrease in viability of DC as assayed by AnnexinV /PI staining with flow-cytometry up to $72 \mathrm{~h}$ after irradiation. Representative result for 1 of 3 donors tested.

Figure 3. FB are functionally resistant to IR. Irradiation of FB did not impair their ability to up-regulate IL-23 secretion from non-irradiated DC; $\mathbf{A}$ - shows a representative donor, $\mathbf{B}$ - summary of 3 donors. C - PGE 2 secretion (determined by ELISA) by FB stimulated with recombinant TNF $\alpha$ and IL $1 \beta(1 \mathrm{ng} / \mathrm{mL})$ is unaffected by IR; C - BJ6 cell line and Drepresentative results for 1 of 2 primary FB donors. E - IR (6Gy) does not affect FB viability up to $24 \mathrm{~h}$ after irradiation as assessed by AnnexinV/PI staining; representative data from 3 experiments.

Figure 4. Mechanisms of IL-23 recovery from irradiated DC by FB. FB-dependent IL23 secretion from irradiated DC is mediated by soluble factors and was independent of cell-cell contact. A - Shows fold increase in IL-23 secretion by DC separated from FB with Transwell $(0.3 \mu \mathrm{m})$ treated with IR (summary of 3 donors). B - Addition of $\mathrm{PGE}_{2}$ to irradiated DC up-regulates IL-23 secretion (summary of 5 donors). C - Up-regulation of IL23 secretion from irradiated DC by FB is COX2 dependent. FB were treated with Indomethacin for $24 \mathrm{~h}$ prior to (and throughout) co-culture with irradiated DC (data from 3 experiments). D - FB did not affect ATM phosphorylation in irradiated DC. Irradiated mDC cultured with FB (blue line) or without FB (orange line) show similar levels of ATM phosphorylation. In both these settings, IR increased ATM activation when compared with non-irradiated mDC (red line) as demonstrated by intracellular staining for flow-cytometry at 2 and $12 \mathrm{~h}$ after IR (6Gy, representative donor of 3). E - Stimulation of TLR4-activated 
DC with the c-AMP agonist forskolin immediately following exposure to IR increased IL-23 secretion (data from 3 representative donors.)

Figure 5. Model of DC-FB crosstalk governing IL-23 dependent Th17 responses after irradiation.

A: TLR-activation of DC in mono-culture elicits secretion of IL-1 $1 \beta,-6$ and -23 which prime Th17 responses. B: The addition of FB to DC provides an important feedback loop that serves to enhance IL-23 secretion and thus augments Th17 responses. C: Irradiation of DC mono-cultures selectively inhibits IL-23 secretion. D: However, the presence of FB ensures that irradiated DC continue to secrete sufficient IL-23 to generate Th17 responses. Importantly, the irradiation of FB does not hinder their reinforcement of IL-23 responses. 
A

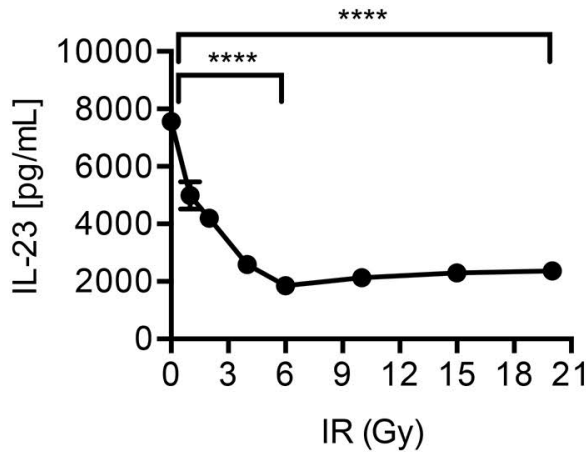

D

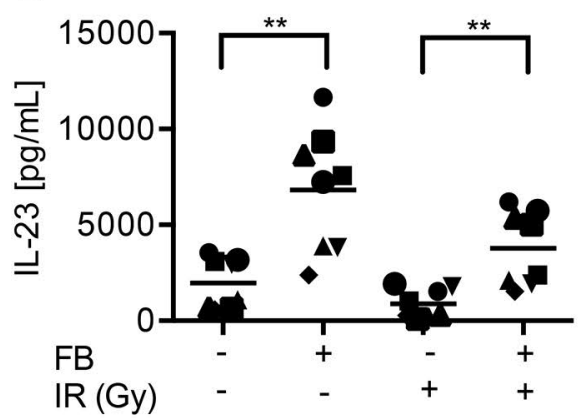

F

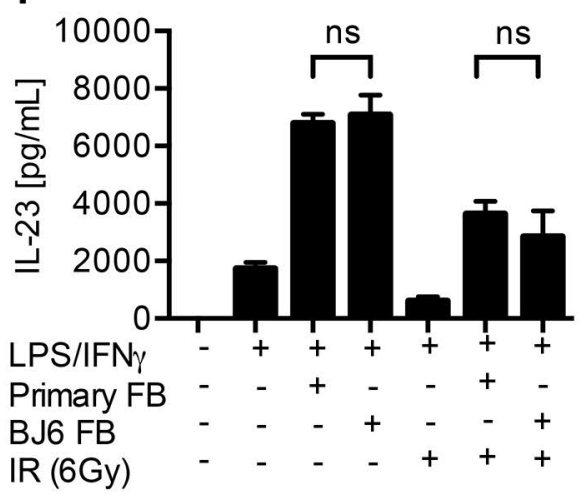

B

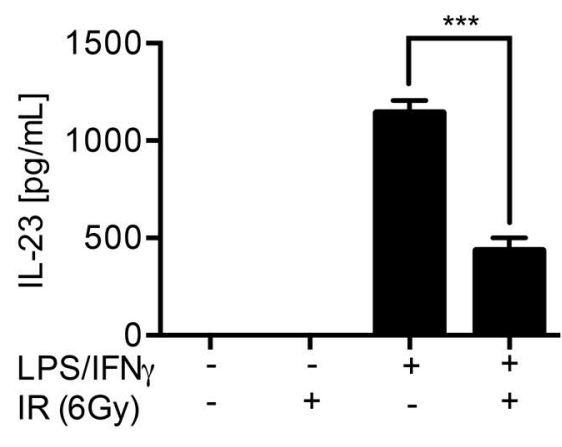

E

G
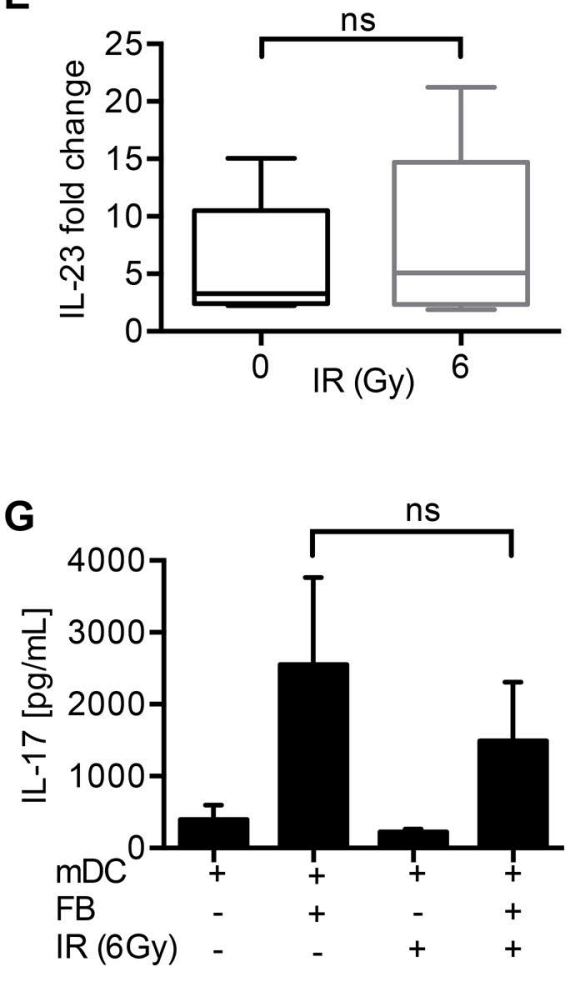

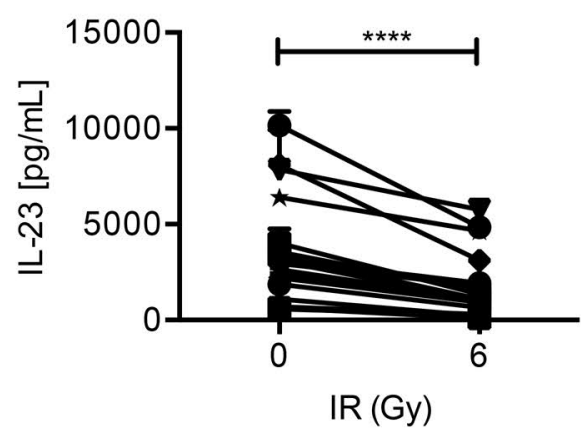

IR (Gy) 


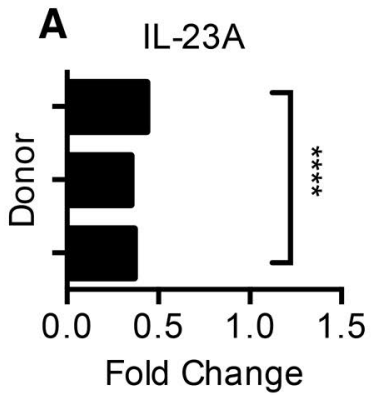

$24 h$

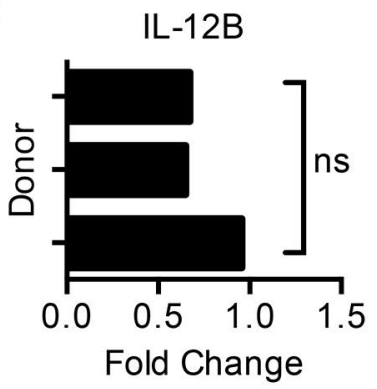

$72 \mathrm{~h}$

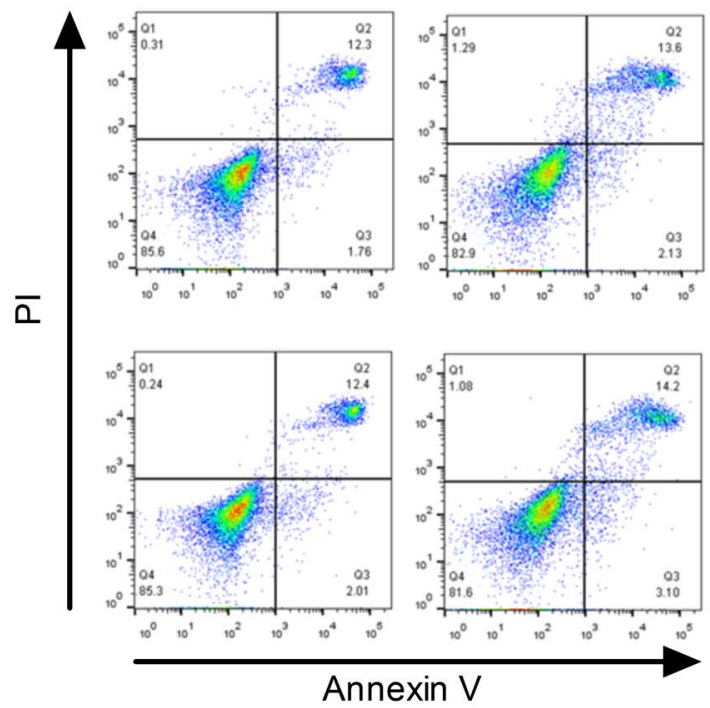

OGy

6Gy 

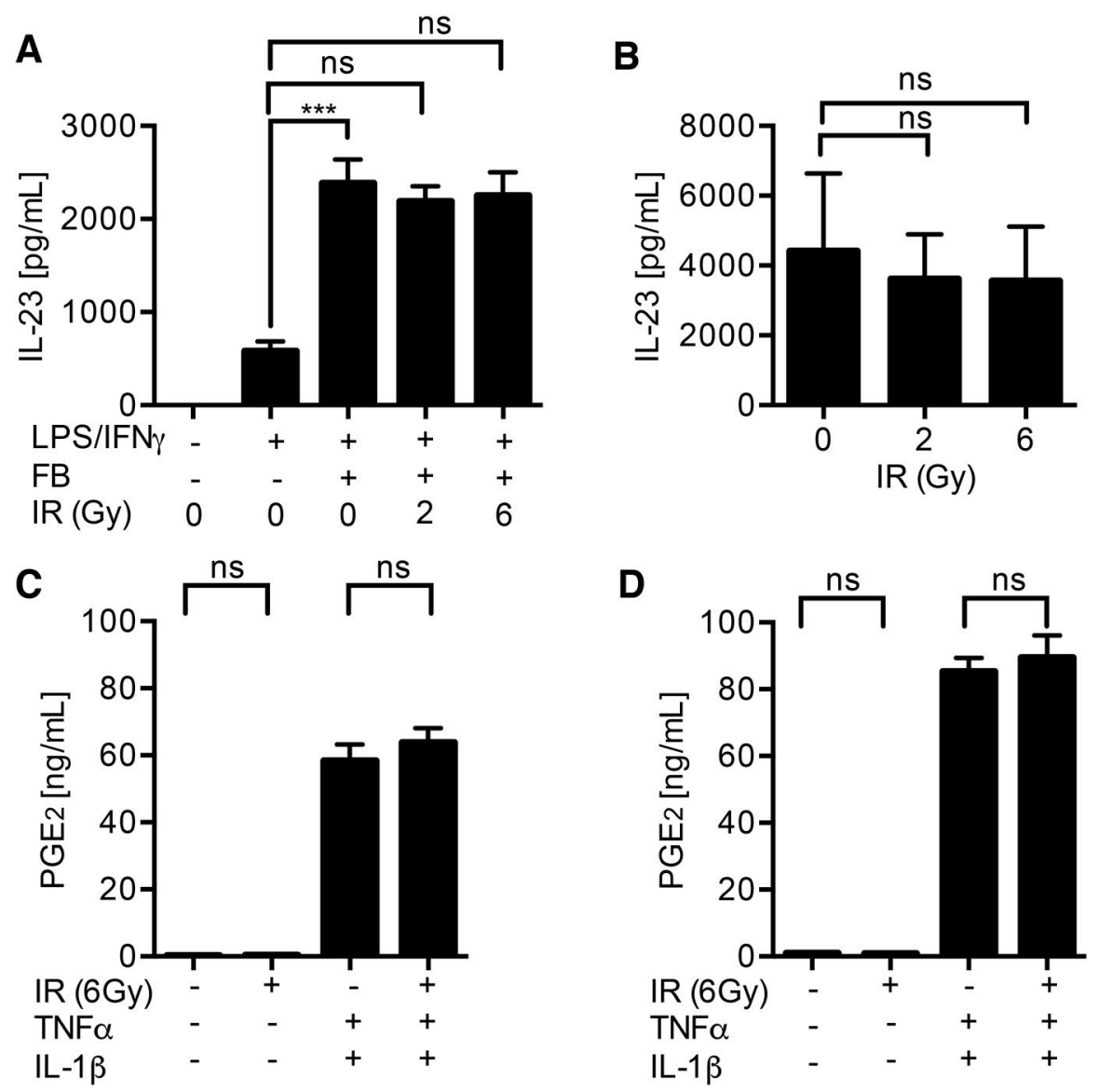

E

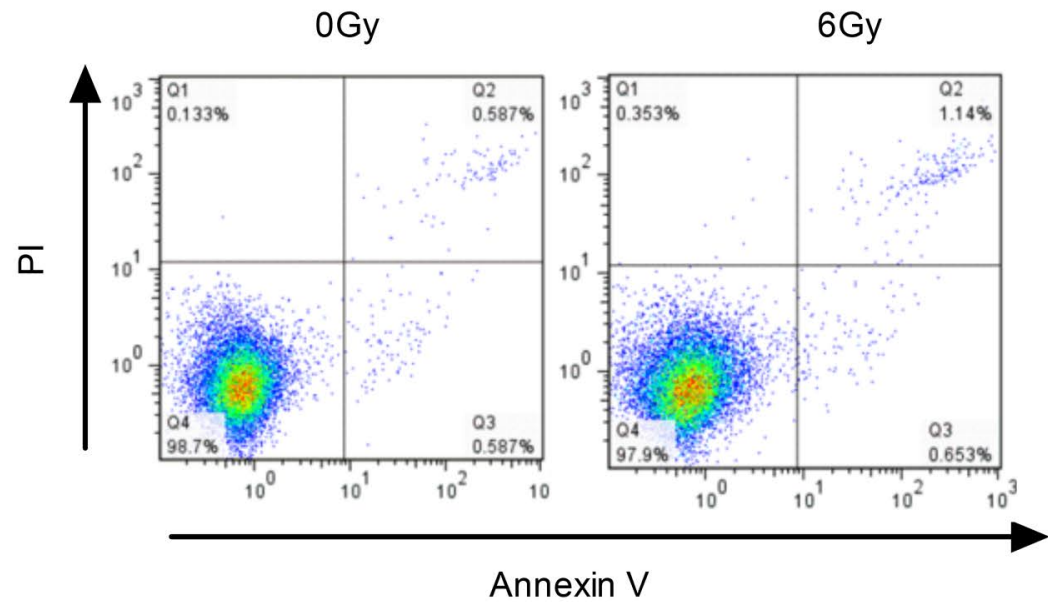


A

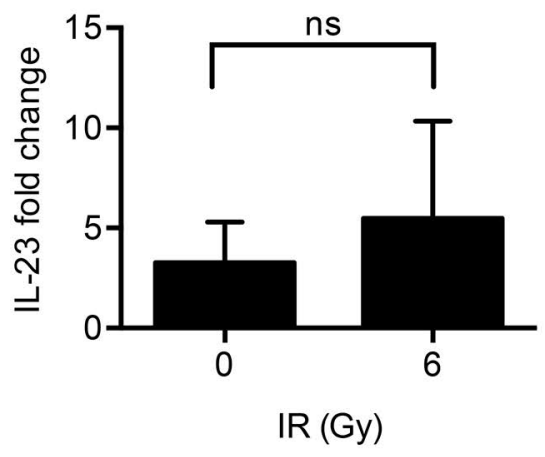

D

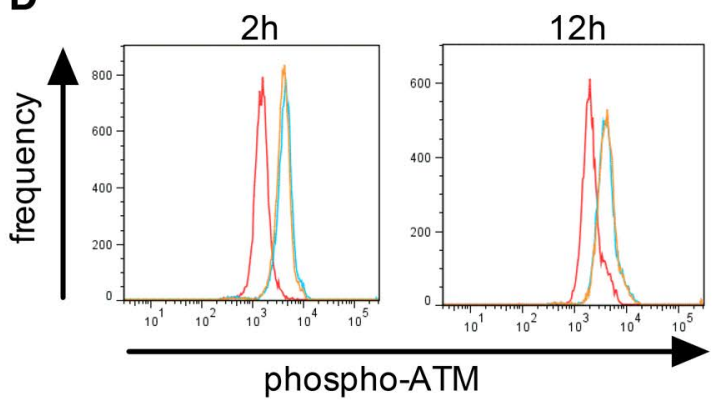

B

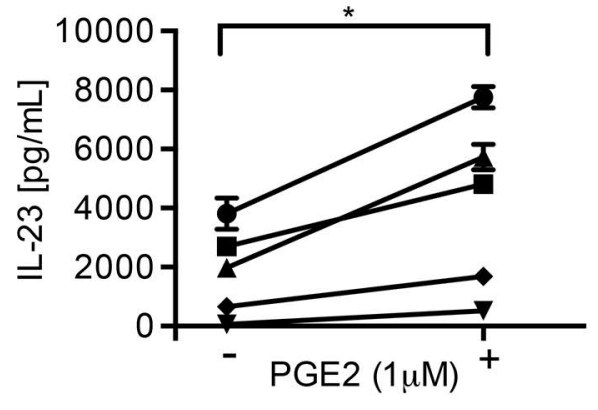

C

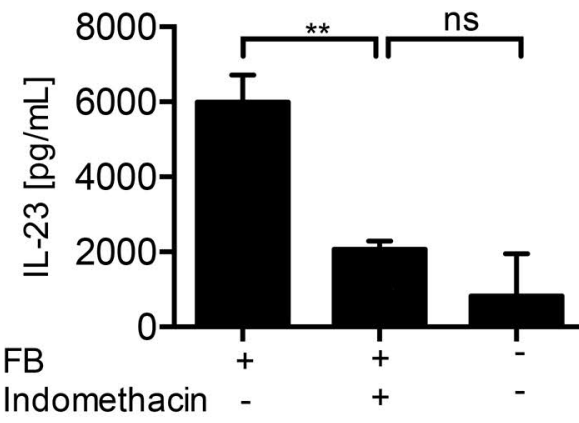

E

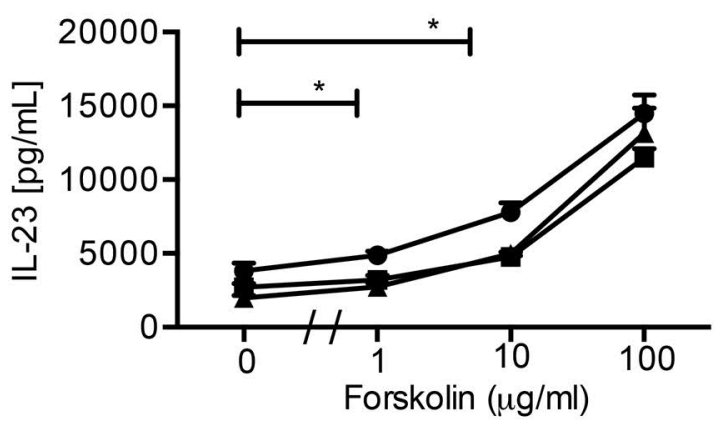


A: DC mono-culture

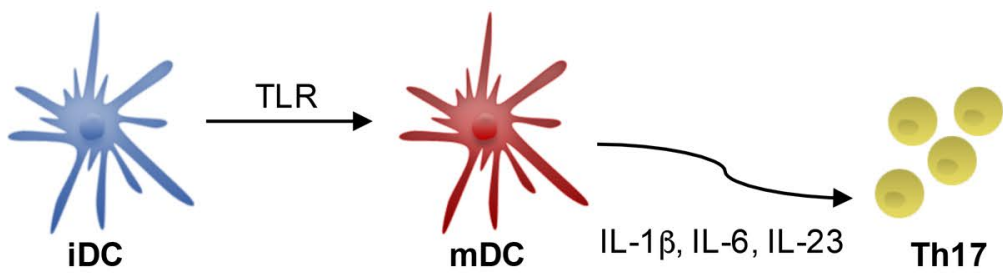

B: DC-FB co-culture

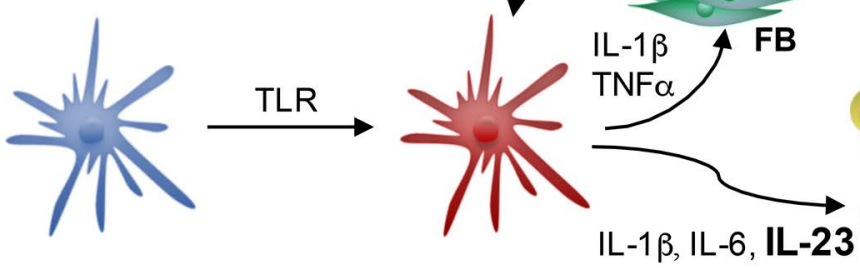

C: Irradiated DC mono-culture

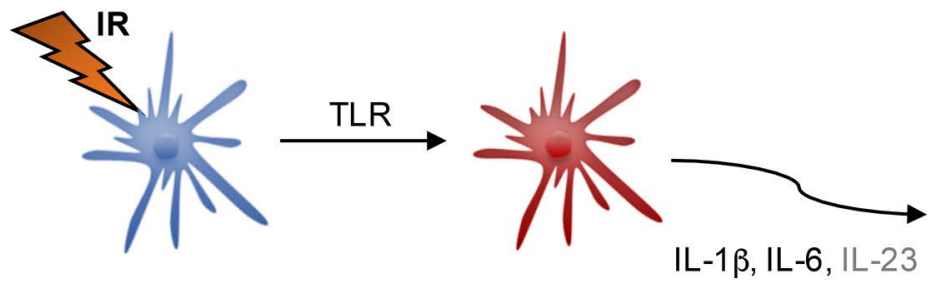

D: Irradiated DC-FB co-culture
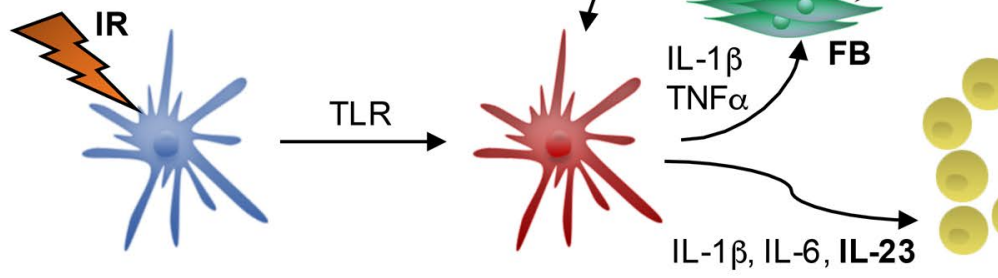
Table 1.

\begin{tabular}{|c|c|c|c|}
\hline Cytokine & $\begin{array}{c}\text { \% remaining } \\
\text { secretion }\end{array}$ & SD & P value \\
\hline IL-23 & 43 & 11 & $<0.0001$ \\
\hline IL-12 & 89 & 16.3 & 0.0005 \\
\hline IL-27 & 100.9 & 36.6 & ns \\
\hline IL-1 $\beta$ & 93.5 & 34.4 & ns \\
\hline TNF $\alpha$ & 111.8 & 29.6 & ns \\
\hline IL-6 & 174.1 & 114.4 & ns \\
\hline IL-10 & 95.9 & 6.2 & $n s$ \\
\hline
\end{tabular}

